\title{
Multi-View Active Learning Optimization Based on Genetic Algorithm and Gaussian Mixture Models for Hyperspectral Data
}

Nasehe Jamshidpour, Student Member, IEEE, Abdolreza Safari, Saeid Homayouni, Senior Member, IEEE

6

Abstract-In this paper, we proposed a novel optimal view generation framework based on the genetic algorithm (GA) and Gaussian mixture models (GMMs) to improve multi-view active learning (MV-AL). AL methods enlarge training data sets, by iteratively selecting the most informative samples, in order to improve classification performance. By using multiple views 10 to build multiple classifiers, the information content of each unlabeled samples can be more accurately estimated. The MV11 AL methods are more inherently suitable for high dimension data such as hyperspectral images. This hybrid framework 12 simultaneously constructs the optimal number of diverse and sufficient views. The proposed algorithm has two main steps. In 13 the first step, by applying a cluster distortion function-based GMMs, the actual number of available independent views is 14 determined. In the next step, a hybrid GA approach selects the optimal combination of views using two different criteria. The 15 experiments were conducted on two benchmark hyperspectral datasets, namely KSC and Indian Pines AVIRIS. The results 16 demonstrated increasing in diversity, and sufficiency of the views compared to the traditional view generation methods. 17 Furthermore, the performance of MV-AL has also been significantly improved.

Index Terms-Active learning (AL), Gaussian Mixture Models (GMMs), Genetic algorithms (GA), Multi-view learning, View generation methods.

(Corresponding author: Abdolreza Safari)

24 N. Jamshidpour and A. Safari are with the School of Surveying and Geospatial Engineering, College of Engineering, University of Tehran, Tehran, Iran. (E-mail: 25 njamshidpour@ut.ac.ir, asafari@ut.ac.ir).

26 S. Homayouni is with the Centre Eau Terre Environnement, Institut National de la Recherche Scientifique, Quebec, Canada (E-mail: saeid.homayouni@ete.inrs.ca). 


\section{INTRODUCTION}

29 The hyperspectral image is a high dimensional data cube consists of many narrow spectral bands. This high dimensionality imposes 30 some difficulties for data processing such as overfitting, inaccurate model's parameters estimation, and the Hughes phenomenon.

31 To overcome these issues highly sophisticated dimension reduction methods have been suggested so far [1, 2].

32 However, this high dimensionality makes them intrinsically suitable for multi-view (MV) [3] for supervised learning algorithms.

33 However, there is also some successful unsupervised multi-view learning method such as [4]. Using multiple views, instead of a

34 single view, for training a learning algorithm, can simultaneously reduce the generalization error rate and improve the learning 35 performance. In addition to dividing the initial feature space into two or more subsets, the curse of dimensionality of the data can 36 be significantly alleviated. However, MV learning methods, for being successful, need an efficient view generation method that 37 produces sufficient and diverse views simultaneously [5]. In other words, each view must be sufficient to train a suitable and 38 reliable classifier and also provide redundant information to solve the classification problem.

39 Active Learning (AL) has recently attracted much attention because of its outstanding performance to improve classification task 40 with a limited number of labelled training data [6-10]. AL methods try to build a compact and well-chosen training data set by 41 selecting the most informative samples for the current model at each iteration [11]. The selected instances are then labeled by an 42 expert and added to the training set. The key difference between various AL methods is the type of query function, which estimates 43 the amount of information content for all candidate samples.

44 The main concern of multi-view active learning (MV-AL) methods is how to choose the most valuable instances using multiple 45 views of the original data. Although, many MV-AL methods have been proposed for hyperspectral image classification [12, 13]. 46 They mostly used simple band partitioning methods based on the correlation between different, such as k-means, correlation, and 47 uniform band slicing. In this way, the sufficiency of each view was not considered. Therefore in this paper, we suggest a hybrid 48 view generation method for hyperspectral data based on the genetic algorithm (GA) that maximize diversity between views and 49 sufficiency of each one.

50 GA is one of the evolutionary optimization algorithms that has been extensively employed to solve multi-criteria optimization 51 problems [14]. The algorithm initializes with a random population which represents the possible solutions to the problem. Then, 52 by the progress of the algorithm, the solution is to approach the optimal answer by considering the criteria.

53 In the current optimization problem, we have dual criteria, first maximizing the sufficiency of each view, and second, minimizing 54 the dependency between views. Thus, by integrating these criteria, a hybrid optimization problem is formed, because the first 55 criteria is a wrapper and the second one is a filter problem. The wrapper methods need to perform the classification is each time, 56 whereas the filter method is independent of the classification task. Therefore, the filter methods are faster, but the wrapper methods 
57 produce a more model-based solution. The obtained experimental results demonstrated the efficiency of the hybrid solution in 58 comparison of wrapper or filter methods.

59 The optimal number of views is another crucial issue in MV-AL which has been mostly neglected in the literature. Although in 60 the earliest MV-AL methods, e.g., Co-EM [15], only two distinct views have been employed, the data structure might imply a 61 different number of views. In this paper, we initially determined the optimal number of views using Gaussian mixture models 62 (GMM) [16]. Secondly, the optimal views by considering the proposed new hybrid criterion were chosen.

\section{Methodology}

\section{A. Finding the Optimal Number of Views}

65 Gaussian mixture models (GMM) are employed to approximate non-gaussian and discrete variables by the weighted sum of a finite 66 number of Gaussian mixture components [17].

$$
P(x \mid \lambda)=\sum_{i=1}^{M} \omega_{i} g\left(x \mid \mu_{i}, \Sigma_{i}\right)
$$

68 where $\mathrm{x}$ is the approximated input D-dimension data vector, $\omega_{i}$ and $g\left(x \mid \mu_{i}, \Sigma_{i}\right)$ are the mixture weight and the Gaussian density, 69 respectively. To approximate the input feature vector, the GMM parameter set $\lambda=\left(\omega_{i}, \mu_{i}, \Sigma_{i}\right)$ must be estimated, with the 70 constraint $\sum_{i=1}^{M} \omega_{i}=1$. There are different popular methods such as maximum likelihood (ML) and expectation maximization 71 (EM) [18]. In this paper, we used the ML method to solve the GMM approximation.

72 The number of these components must be determined as a prior which significantly affects the emerged components. Therefore, 73 some studies are trying to find the optimal number of the components $[19,20]$ that also indicate the number of available clusters. 74 Incremental $k$-means clustering is one of the mixtures' number optimization method [20]. In this method, $k$ is gradually increased 75 as long as all components are still independent. The mutual information is employed for determining the statistical independence. 76 If by adding the $k^{\text {th }}$ component, at least one component becomes dependent on the other components, the optimal number of 77 components will be chosen as $(k-1)$.

78 Another way to estimate the optimal number is the cluster distortion function-based approach [19]. In this paper, we used this 79 algorithm which proposes the function $f(k)$ based on the sum of cluster distortion $\left(I_{j}\right)$ for $k$ number of clusters:

$$
S_{k}=\sum_{j=1}^{k} I_{j}
$$

81 The function $f(k)$ is defined as the ratio of $S_{k}$ and $S_{k-1}$ as follows:

$$
f(k)= \begin{cases}1 & k=1 \\ \frac{s_{k}}{\alpha_{k} s_{k-1}} & k>1\end{cases}
$$

83 The coefficient $\alpha_{k}$ is also defined by the coefficient value of the previous step $\alpha_{k-1}$ : 


$$
\alpha(k)= \begin{cases}1-\frac{3}{4 D} & k=2 \\ \alpha_{k-1}+\frac{1-\alpha_{k-1}}{6} & k>2\end{cases}
$$

85 and $\mathrm{D}$ is the dimension of the data vector. The minimum value of $f(k)$ indicates the most concentrated clustering and also the 86 optimal number of components. We used the incremental search of $k$ to minimize the $f(k)$ function.

\section{B. View Generation by Hybrid GA-FSS Method}

88 The main characteristic of MV-AL algorithms is how to generate views. The constructed views should have some essential 89 characteristics to ensure the superiority of the MV-AL over single view AL methods.

90 First, each view must impose redundant information compared to the other views, which is measured by the diversity of the views.

91 Moreover, the views should be as independent as possible from each other. On the other hand, the sufficiency of each view to 92 building a good classifier should be taken into account. Our proposed GA-based view generation method tries to find the best set 93 of multiple-views by integrating all the above criteria. The general framework of the proposed GA-based method is given in Fig. 941.

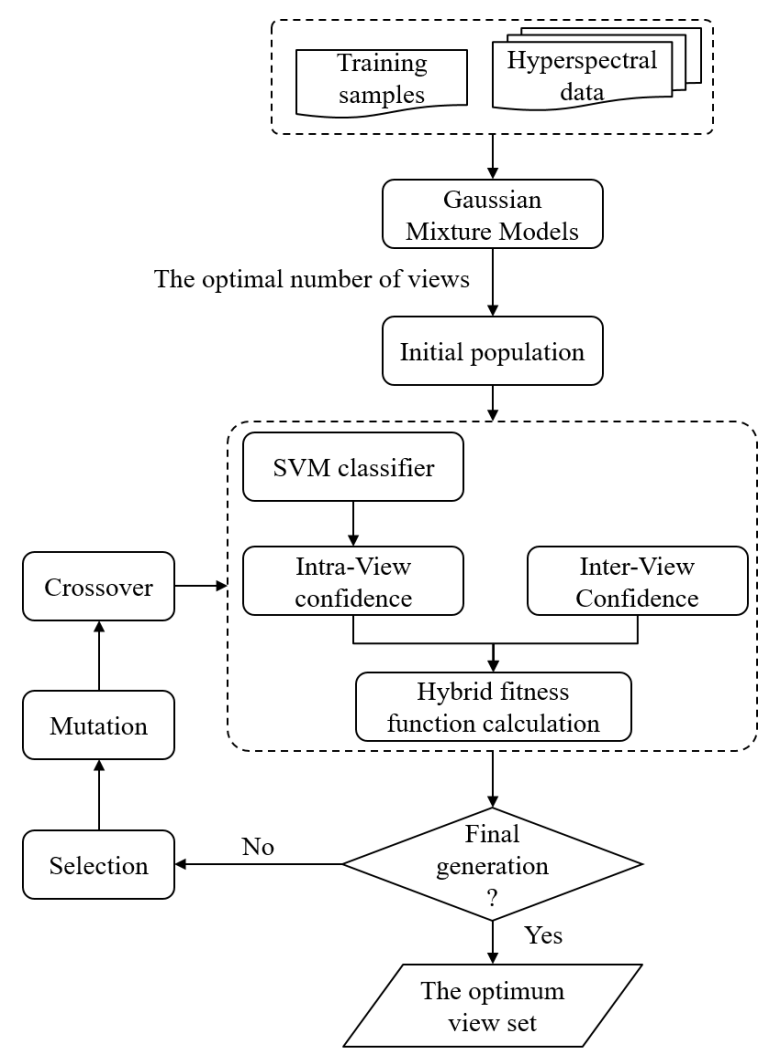

Fig. 1. Flowchart of the proposed hybrid GA-FSS method. 
111 GA has been mostly used as a feature subset selection (FSS) method for dimension reduction of hyperspectral data [21]. The

112 primary goal of FSS is to build a sufficiently unique and compact subset of features. We proposed a novel framework based on

113 GA-FSS to construct multiple diverse, dependent and sufficient views. The FSS problem traditionally is solved in two ways using

114 either wrapper or filter criteria. To find out a more appropriate solution, we employed a hybrid approach by integrating both filter

115 and wrapper criteria. For this purpose, we introduce the inter-confidence criteria representing the diversity and independence

116 between all the views, as the filter method:

$$
C_{\text {inter }}=\sum_{i=1}^{N_{v}} \frac{1}{M I\left(X^{i}, X^{j}\right)}
$$

$118 M I\left(X^{i}, X^{j}\right)$ is the mutual information between the $i^{\text {th }}$ and $j^{\text {th }}$ views. In the other hand, we used the intra-confidence as the wrapper 119 criteria, which is computed by the mean of all views' sufficiency.

$$
C_{\text {intra }}=\frac{1}{N_{v}} \sum_{i=1}^{N_{v}} P\left(X_{L}^{i}, X_{U}^{i}, Y_{L}\right)
$$

121 where $\mathrm{P}$ represents the classification performance. Therefore, the hybrid fitness function is formed by the weighted summation of 122 both filter and wrapper criteria using $w_{f}$ and $w_{w}$ weights respectively.

$$
F=w_{f} * C_{\text {inter }}+w_{w} * C_{\text {intra }}
$$

125 The weights are experimentally set as $w_{f}=w_{w}=0.5$ using $2 \mathrm{D}$ grid search method with the search space $\{0.1,0.2,0.3, \ldots, 0.9\}$ 126 for each parameter.

127 In the GA algorithm, each chromosome represents a solution, and at each generation, the population gets closer to the optimum 128 solution by evolutionary operators such as selection, crossover and mutation and migration. Therefore, in our proposed GA scheme 129 a binary chromosome with the size of $N_{v} \times D$ was used, where D and $N_{v}$ are the initial dimension of hyperspectral data and number 130 of views. Each D bits represent a single view, where the value of 0 or 1 for each bit shows the presence or absence of the 131 corresponding band in the view.

\section{EXPERIMENTAL RESULTS}

\section{A. Hyperspectral Data}

134 For the evaluation of the proposed method, two hyperspectral data set, provided over the Kennedy Space Center (KSC) and Indian

135 Pines, were employed. These data sets, acquired by the AVIRIS sensor, but with different 18-m and 30-meter spatial resolutions 136 due to their different altitude. After excluding noisy and water absorption bands, 176 and 185 bands remain for KSC and Indian 137 Pines dataset, respectively. The KSC image contains 512×614 pixels which include 13 different land cover classes with overall 
1385,211 labelled samples. While the Indian Pines data set including 16 different land cover classes with 10,366 ground truth samples 139 in a 145 by 145 scene.

\section{B. Experimental Setup}

141 The experiment was conducted using the training datasets that were produced by combining ' 5 -fold' and 'hold-out' randomly 142 selected cross-validation schemes to make experimental results robust to the training sets and prove the generalization ability of 143 the proposed model. First, the initial labelled data set was divided into 5-fold complementary subsets. Then, the 'hold-out' cross144 validation mechanism was employed. We have considered the worst scenario with the smallest size of the training set, i.e. only 145 five labelled samples per class. The performance of the proposed and baseline methods was evaluated using the average overall 146 accuracy $(\overline{O A})$ over five folds.

147 Support vector machine (SVM) was used as the classifier because of its excellent performance and it only two free parameters that 148 need to be tuned. They were tuned using 2-D grid search with a search space of $0.05,0.1,0.15, \ldots, 0.95$.

149 At the first iteration of the proposed MV-AL framework, the SVM classifier was trained, then by using the AMD query function 150 [12] the five most informative samples were added at each step. The AL algorithm stopped after reaching to 40 iterations and 151 adding 200 samples to the training dataset.

152 There are also some parameters of GA which must be selected. The initial population was randomly generated with a size of 50 153 individuals. The crossover, migration and mutation rate were selected as $0.8,0.2$ and 0.05 respectively. The elitism selection 154 strategy was implemented. The maximum number of generation was set to 100 , and the number of consecutive unchanged iterations 155 was 20

\section{$156 \quad$ C. Experimental Results}

157 In this paper, two experiments were conducted to investigate the efficiency of our proposed method. First, we determined the 158 maximum number of the available independent views in the datasets using GMMs. In the second experiment, the GA-FSS view 159 generation was compared with six related methods.

160 The optimal number of independent views was estimated using the proposed cluster distortion function-based GMMs method. The 161 experimental results for both datasets are reported in Table I.

162 As it can be seen for each data set, there is an optimal number of views with the minimum value of the cluster distortion function $163 f(k)$. Also, to evaluate the obtained views, we computed the average mutual information $(\overline{M I})$ between each pair of views. As 164 shown the optimal number of views provides the most diverse views with the minimum averaged mutual information value. On 165 the other hand, the required CPU time by the proposed hybrid GA-FSS method are reported in the table. As expected, by increasing 
166 the number of views the computation cost also increases. Therefore, employing the proposed function-based GMMs method to

167 find the optimal number of views is an essential and affordable preliminary step for MV-AL.

TABLE I

CLUSTER DISTORTION FUNCTION $(f(k))$, MUTUAL INFORMATION $(\overline{M I})$ AND REQUIRED CPU TIME (TIME) FOR THE DIFFERENT NUMBER OF GENERATED VIEWS BY THE PROPOSED HYBRID GA-FSS METHOD.

\begin{tabular}{|c|c|c|c|c|c|c|}
\hline \multirow{2}{*}{$\begin{array}{l}\text { Number of } \\
\text { views }\end{array}$} & \multicolumn{3}{|c|}{ KSC } & \multicolumn{3}{|c|}{ Indian Pines } \\
\hline & $f(k)$ & $\overline{\mathrm{MI}}$ & Time (s) & $f(k)$ & $\overline{\mathrm{MI}}$ & Time (s) \\
\hline 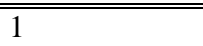 & 1.00 & $\begin{array}{l}----- \\
\end{array}$ & "----- & 1.00 & $\begin{array}{l}---- \\
--\end{array}$ & $\begin{array}{l}---- \\
--\end{array}$ \\
\hline 3 & 0.98 & 0.317 & 443.89 & 1.04 & 0.286 & 578.96 \\
\hline 4 & 0.32 & 0.109 & 523.86 & 0.31 & 0.591 & 715.48 \\
\hline 5 & 1.39 & 0.298 & 558.88 & 1.26 & 1.105 & 846.73 \\
\hline
\end{tabular}

169 We also investigated the effect of the number of views on MV-AL performance. The proposed GA-FSS method generated a 170 different number of views. The learning curves of the performed MV-AL on these sets of views for KSC data set are given in Fig. 171 2. The best performance was achieved by the four views which have been already determined as the optimal number by our 172 proposed method.

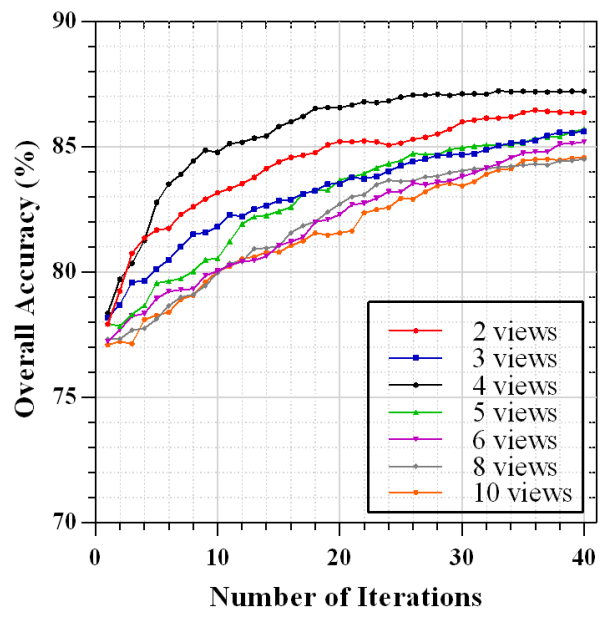

Fig. 2. Average learning curves of MV-AL for the different number of views using the proposed GA-FSS view generation method for KSC dataset.

175 In the second experiment, to demonstrate the effectiveness of the proposed GA-hybrid view generation method, the obtained results 176 were compared to four traditional and also two other GA methods. The baseline methods were conventional band clustering 177 algorithms including k-means, correlation, uniform, and random view generation methods which were proposed in [12]. Two 178 single criterion GA-based methods were also implemented, a wrapper method as called GA-intra which only considers the intra179 confidence. The other one was GA-inter method which is a filter method because of its independence of the classifier performance. 
180 As can be seen in Fig. 3, the learning curve of the proposed method has the best performance in the KSC data set. As expected, 181 random band clustering had the worst performance, while other band clustering methods produced similar results to some extent.

182 Also, the numerical results of the proposed view generation methods and two state-of-the-art methods, view updating (VU) [12] 183 and multiple morphological component analysis (MMCA) [13] for both KSC and Indian Pines data sets are presented in Table II.

TABLE II

ACHIEVED AVERAGEd OVERALl ACCURACY $(\overline{\mathrm{OA}})$ AFTER 40 ITERATIONS OF MV-AL METHOD AND REQUIRED CPU TIME BY DIFFERENT VIEW GENERATION METHODS FOR KSC AND INDIAN PINES DATASETS.

\begin{tabular}{|c|c|c|c|c|}
\hline \multirow{2}{*}{ VG Method } & \multicolumn{2}{|c|}{ KSC } & \multicolumn{2}{|c|}{ Indian Pines } \\
\hline & $\overline{\mathrm{OA}}(\%)$ & Time (s) & $\overline{\mathrm{OA}}(\%)$ & Time (s) \\
\hline K-Means & 85.50 & 11.31 & 81.76 & 15.64 \\
\hline GA-inter & 86.14 & 420.34 & 82.28 & 312.77 \\
\hline VU [12] & 85.63 & 356.12 & 82.01 & 298.47 \\
\hline MMCA [13] & 85.47 & 207.98 & 82.63 & 143.28 \\
\hline GA-hybrid & 87.21 & 523.86 & 83.93 & 498.53 \\
\hline
\end{tabular}

Although the proposed hybrid view generation method implied a higher computation cost in comparison to the other methods, it

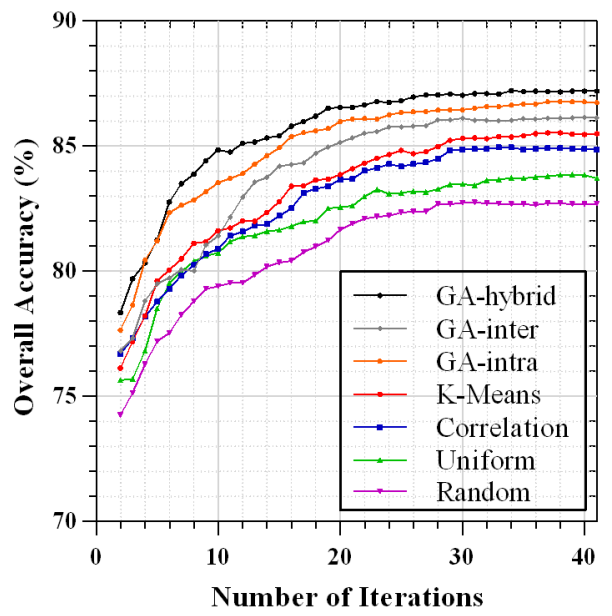

Fig. 3. Average learning curves of MV-AL for different view generation methods using four views and 5-fold cross-validation for KSC dataset.

\section{CONCLUSION}

192 In this paper, we designed a novel framework to produce an optimal multi-view set to enhance hyperspectral multi-view active 193 learning performance. The framework consisted of two steps. In the first step, the actual number of underlying independent views 
194 in the data was determined by GMMs. Then, a hybrid view generation method based on GA produced the optimal view set by 195 considering two different filter and wrapper criteria. The filter criterion was defined to maximize the diversity between each pair

196 of views, while the wrapper criterion tried to guarantee the sufficiency of the views for learning the classifier. The experiments 197 have been conducted on KSC-AVIRIS and Indian Pines datasets. The experimental results confirmed that the proposed GMM 198 function-based method efficiently selects the best number of views. By employing this method both the view intensity and the 199 performance of the MV-AL improved. Therefore, the low computation cost and the observed efficiency of this method make it 200 suitable as a basic step in all MV algorithms. On the other hand, a significant improvement achieved by the proposed GA-based 201 view generation method compared to the conventional and also single-criterion GA-based methods. Despite the expected high 202 computation cost of the proposed method, it was able to enhance the diversity and sufficiency of the views and also the final MV203 AL method, consequently.

\section{REFERENCES}

[1] X. Jia, B.-C. Kuo, and M. M. Crawford, "Feature mining for hyperspectral image classification," Proceedings of the IEEE,

[2] Q. Wang, F. Zhang, and X. Li, "Optimal Clustering Framework for Hyperspectral Band Selection," IEEE Transactions on Geoscience and Remote Sensing, pp. 1-13, 2018.

[3] C. Xu, D. Tao, and C. Xu, "A survey on multi-view learning," arXiv preprint arXiv:1304.5634, 2013.

[4] Q. Wang, M. Chen, F. Nie, and X. Li, "Detecting Coherent Groups in Crowd Scenes by Multiview Clustering," IEEE transactions on pattern analysis and machine intelligence, 2018. categorization," Expert Systems with Applications, vol. 49, pp. 31-47, 2016. Image Classification," IEEE Transactions on Geoscience and Remote Sensing, 2017.

[9] B. Du, Z. Wang, L. Zhang, L. Zhang, W. Liu, J. Shen, et al., "Exploring representativeness and informativeness for active learning," IEEE transactions on cybernetics, vol. 47, pp. 14-26, 2017. 
223 [10] Y. Gal, R. Islam, and Z. Ghahramani, "Deep Bayesian Active Learning with Image Data," arXiv preprint $224 \quad$ arXiv:1703.02910, 2017.

225 [11] D. Tuia, M. Volpi, L. Copa, M. Kanevski, and J. Munoz-Mari, "A survey of active learning algorithms for supervised remote sensing image classification," Selected Topics in Signal Processing, IEEE Journal of, vol. 5, pp. 606-617, 2010.

[12] W. Di and M. M. Crawford, "View generation for multiview maximum disagreement based active learning for 229 hyperspectral image classification," Geoscience and Remote Sensing, IEEE Transactions on, vol. 50, pp. 1942-1954, 2012.

[13] X. Xu, J. Li, and S. Li, "Multiview Intensity-Based Active Learning for Hyperspectral Image Classification," IEEE Transactions on Geoscience and Remote Sensing, vol. 56, pp. 669-680, 2018.

A. P. Engelbrecht, "Introduction to Evolutionary Computation," in Computational Intelligence: An Introduction, 2nd ed Chichester: John Wiley \& Sons, Ltd, 2007, pp. 127-142. W. Wang and Z.-H. Zhou, "Analyzing co-training style algorithms," in European Conference on Machine Learning, 2007, pp. $454-465$.

[18] S. Bonhomme, K. Jochmans, and J. M. Robin, "Non-parametric estimation of finite mixtures from repeated measurements," Journal of the Royal Statistical Society: Series B (Statistical Methodology), vol. 78, pp. 211-229, 2016. load flow study," in India Conference (INDICON), 2016 IEEE Annual, 2016, pp. 1-6.

D. Reynolds, "Gaussian mixture models," Encyclopedia of biometrics, pp. 827-832, 2015. 